\title{
Medicina de desastres: una necesidad
}

\author{
Luis D. Sánchez-Arreola ${ }^{1,2 *}$ y Elisa N. Saleme-Cruz ${ }^{2}$ \\ ${ }^{1}$ Departamento de Urgencias, Hospital General La Villa, Secretaría de Salud; ${ }^{2}$ Departamento de Urgencias, Hospital Centro Médico ABC Santa \\ Fe. Ciudad de México, México
}

\section{Resumen}

A lo largo de la historia, la naturaleza se ha encargado de recordar a los humanos su enorme y sorpresivo poder destructivo. México no es la excepción y estas experiencias han servido de parteaguas para la conformación del Sistema Nacional de Protección Civil y Gestión de Riesgos que opera en la actualidad. Desde hace más de 20 años, la Organización de las Naciones Unidas ha prestado especial atención a la reducción de los desastres por diferentes vías y mediante el trabajo colectivo. En nuestro país destaca la ausencia, en los planes de estudios y programas operativos de los especialistas en medicina de urgencias, de una formación específica, especializada y metodológica en medicina de desastres.

Palabras clave: Desastres. Emergencias mayores. Medicina de desastres. Catástrofes.

\section{Disaster medicine: a must}

\begin{abstract}
Throughout history, nature has overseen reminding humans of its enormous and surprising destructive power. Mexico is no exception and these experiences have served as a watershed for the creation of the National System of Civil Protection and Risk Management that currently operates. For more than 20 years, the United Nations Organization has paid special attention to disaster reduction in different ways and through collective work. In our country, the absence in the curricula and operational programs of emergency medicine specialists of a specific, specialized and methodological training in disaster medicine stands out.
\end{abstract}

Keywords: Disasters. Major emergencies. Disaster medicine. Catastrophes.

A lo largo de la historia, la naturaleza se ha encargado de recordar al hombre su enorme y sorpresivo poder destructivo. Sismos, erupciones volcánicas, inundaciones, huracanes y los recientes eventos epidemiológicos como la pandemia de COVID-19 son ejemplos que ponen al descubierto la vulnerabilidad del ser humano y la necesidad de estar mejor preparados para disminuir los efectos y el impacto negativo de estos fenómenos sobre la salud pública.

México no es la excepción, pues tan solo en los últimos 20 años nuestro país ha resultado mayormente afectado por diversos fenómenos naturales, emergencias mayores, crisis sanitarias y fenómenos socio-organizativos que en conjunto, hasta el día de hoy, han

\section{Correspondencia:}

*Luis D. Sánchez-Arreola

E-mail: dano23sa@ hotmail.com
Disponible en internet: 14-02-2022 Rev Educ Investig Emer. 2022;4(1):37-39 www.medicinadeemergencias.com

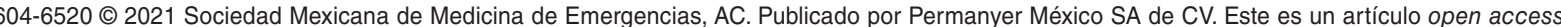
bajo la licencia CC BY-NC-ND (http://creativecommons.org/licenses/by-nc-nd/4.0/). 
ocasionado registros históricos de alrededor de 70,000 víctimas fatales, y que han servido de parteaguas para la conformación del Sistema Nacional de Protección Civil y Gestión de Riesgos que opera en la actualidad ${ }^{1}$.

Debido a lo anterior, desde hace más de 20 años la Organización de las Naciones Unidas ha prestado una especial atención a la reducción de los desastres por diferentes vías y mediante el trabajo colectivo de diversos actores, siendo de especial importancia la declaración de la pasada década como el "Decenio Internacional para la Reducción de los Desastres", que logró impulsar en todo el mundo la importancia de la prevención y la mitigación de los desastres, así como la preparación y la respuesta a estos, además de la ayuda a los países para mitigar sus efectos ${ }^{2,3}$.

Sin embargo, es importante comentar que un común denominador en la mayoría de las situaciones de desastre por las que ha pasado nuestro país ha sido el impacto negativo que han tenido en los sistemas de salud y sus componentes, ocasionando desde cuantiosos daños a la infraestructura hospitalaria hasta una verdadera crisis en la operación de los servicios de atención médica debido al colapso de los establecimientos de salud y la sobredemanda de atenciones en situaciones emergentes y extraordinarias, por lo que de este concepto se desprende con mayor énfasis que un desastre no solo es un fenómeno natural o causado por el hombre, sino hace referencia también a las consecuencias y a su impacto sobre una sociedad, una comunidad o un territorio determinados ${ }^{4,5}$.

En alineación con uno de los objetivos de la Estrategia Internacional de las Naciones Unidas para la Reducción de Desastres, «Estimular la investigación y la aplicación, transferir conocimientos, trasmitir experiencia, crear capacidades y asignar los recursos necesarios que permitan reducir o prevenir los efectos graves y reiterados de los peligros para las poblaciones más vulnerables", toma especial relevancia la gran necesidad de contar con profesionales sanitarios preparados no solo para llevar a cabo las acciones de campo y atención médica extrahospitalaria, sino también con profesionales médicos entrenados de forma multidisciplinaria en evaluación de daños, gestión, planeación y operación de los planes de respuesta sanitarios ante las diversas catástrofes, que cuenten con una formación base, sistema de estudio y un ejercicio médico que englobe las disciplinas de la medicina de urgencias y la salud pública en su conjunto, capaces de disminuir el impacto negativo de emergencias mayores y desastres, es decir, verdaderos gerentes de la respuesta de la medicina de urgencias en situaciones catastróficas ${ }^{2,3}$.
En la actualidad, en nuestro país destaca la ausencia, en los planes de estudios y programas operativos de los especialistas en medicina de urgencias, de una formación específica, especializada y metodológica en medicina de desastres que tome como base fundamental a la especialidad de urgencias para complementar esta formación con nuevos conocimientos técnico-profesionales y habilidades relacionadas con situaciones de desastre de toda índole, que permita a estos profesionales adquirir la capacidad de concentrar su conocimiento y esfuerzo en la toma de decisiones para disminuir el impacto negativo en la salud y mejorar las posibilidades de supervivencia con los recursos disponibles. Actualmente solo se imparten programas en formato de cursos cortos o pasantías con aplicación limitada que solo hacen énfasis en la gestión de riesgos desde el plano de la protección civil, dejando atrás la operación y los planes de respuesta sanitarios.

De acuerdo con el análisis efectuado por la Organización Panamericana de la Salud (OPS) sobre la respuesta a múltiples desastres en todo el mundo y en la región de las Américas, queda demostrada la necesidad de contar con profesionales médicos entrenados, pues en la mayoría de los casos la respuesta limitada del sector salud se ha caracterizado por un importante número de profesionales médicos que no estaban preparados o incluso llegaron a ser incompetentes en las acciones de atención médica ante fenómenos que ocasionaron víctimas en masa, acompañados de instituciones de salud y hospitales carentes de recursos y planes de actuación y respuesta en emergencias, por lo que es una necesidad imperiosa la creación de una especialidad anclada a la medicina de urgencias que dicte los principios, los criterios y los estándares para la respuesta de los equipos médicos en casos de emergencia y desastres en alineación con los estándares y la normativa internacionales ${ }^{3}$.

Los especialistas en medicina de desastres deben contar con una formación médica y un entrenamiento en medicina de urgencias que constituyan los pilares fundamentales de la formación profesional, los cuales se complementen con la adquisición de conocimientos técnicos, médicos y operativos centrados en temas tan importantes como la epidemiología de los desastres, la implementación de planes de preparación y respuesta hospitalaria, la gestión de la atención médica de urgencia en incidentes con víctimas en masa, la identificación y el manejo de emergencias epidemiológicas, la gestión y el establecimiento de los hospitales móviles y su operación, la coordinación de los servicios prehospitalarios de primera respuesta, así como el 
apoyo y la contención psicológica de las víctimas y del personal de salud involucrados en un desastre, fortalecidos por un campo clínico con destrezas prácticas específicas $^{6,7}$.

Es importante mencionar que el campo de acción de los futuros especialistas en medicina de desastres proyecta una enorme oportunidad de complementar y robustecer las acciones de los sistemas locales y regionales de salud, así como de las direcciones y coordinaciones médico-operativas de los servicios de emergencia, además de asegurar, en alineación con la iniciativa de la OPS, la misión de desarrollar y contar con equipos médicos de emergencia entrenados y autosuficientes, liderados por profesionales que puedan responder a tiempo y con conocimientos sólidos a las poblaciones afectadas por emergencias y desastres en su propio país, en la región y a nivel global, respaldados por los gobiernos y las organizaciones conforme a un estándar internacional único y en cualquier momento en que sea necesario ${ }^{6}$.

La historia nos ha demostrado que los desastres han formado parte de la vida del hombre desde su creación, por lo que son y serán siempre un acompañante incómodo e inherente a la evolución del hombre. Sin embargo, el paso del tiempo nos ha señalado también que los efectos que genera la humanidad con su interacción con el planeta son, y seguirán siendo, el principal factor de riesgo para sufrir el embate de futuros fenómenos naturales, desastres y emergencias, por lo que está en nosotros entender el riesgo que esto conlleva y estar mejor preparados para enfrentarlo.

\section{Financiamiento}

No existe ningún financiamiento relacionado con esta publicación.

\section{Conflicto de intereses}

Los autores declaran que no existen conflictos de intereses.

\section{Responsabilidades éticas}

Protección de personas y animales. Los autores declaran que para esta investigación no se han realizado experimentos en seres humanos ni en animales.

Confidencialidad de los datos. Los autores declaran que en este artículo no aparecen datos de pacientes.

Derecho a la privacidad y consentimiento informado. Los autores declaran que en este artículo no aparecen datos de pacientes.

\section{Bibliografía}

1. Alcántara-Ayala I. Desastres en México: mapas y apuntes sobre una historia inconclusa. RIG. 2019;(100). 21 de noviembre de 2019. (Consultado 27-12-2021.) Disponible en: http://www.investigacionesgeograficas. unam.mx/index.php/rig/article/view/60025.

2. Organización Mundial de la Salud. A strategic framework for emergency preparedness. Ginebra: WHO Documents Productions Services; 2017. (Consultado 27-12-2021.) Disponible en: https://www.who.int/publications/i/item/a-strategic-framework-for-emergency-preparedness.

3. Organización Panamericana de la Salud. Recomendaciones para la expansión de capacidades de atención clínica y desplieque de equipos médicos de emergencia. Washington; PAHO; 2021. (Consultado 27-122021.) Disponible en: https://iris.paho.org/handle/10665.2/54327.

4. Mejías CT, Mejías CLM, Cabrera RMC, Amador MJL. La medicina de desastres en el plan de estudios del médico general. EduMeCentro. 2015;7:177-87.

5. Mestre CVA, Salabert TI, Dihigo FMT, Hernández BE, Núñez VL, Fagundo MF. La asignatura medicina de desastres: su importancia en la formación curricular de los estudiantes de medicina. Rev Med Electron. 2017;39:615-9.

6. Colectivo de autores. Medicina de desastres. La Habana: Editorial Ciencias Médicas; 2004.

7. Organización Panamericana de la Salud. Manuales y guías sobre desastres: protección de la salud mental en situaciones de desastres y emergencias. Washington: PAHO; 2002. Disponible en: https://www.paho.org/ disasters/dmdocuments/GuiaTecnicaDeSaludMentalEnSituacionesDeDesastresEmergencias.pdf 